Research, Society and Development, v. 11, n. 3, e3911326166, 2022

(CC BY 4.0) | ISSN 2525-3409 | DOI: http://dx.doi.org/10.33448/rsd-v11i3.26166

\title{
Coexistência de tumores estromais gastrointestinais (GISTs), feocromocitoma e paragangliomas em uma paciente com Neurofibromatose tipo 1: Relato de Caso
}

Coexistence of gastrointestinal stromal tumors (GISTs), pheochromocytoma and paragangliomas in a patient with neurofibromatosis type 1: a case report

Coexistencia de tumores del estroma gastrointestinal (GIST), feocromocitoma y paragangliomas en un paciente con neurofibromatosis tipo 1: reporte de un caso

Raiana Carol de Medeiros Dantas ORCID: https://orcid.org/0000-0001-8405-8784 Universidade Federal do Rio Grande do Norte, Brasil E-mail: raianacarol12@hotmail.com

Lucca Ferdinando Queiroz Fernandes

ORCID: https://orcid.org/0000-0002-0640-0588 Universidade Federal do Rio Grande do Norte, Brasil E-mail: queirozlucca@gmail.com

Iago Tavares de Carvalho

ORCID: https://orcid.org/0000-0001-8008-8580 Universidade Federal do Rio Grande do Norte, Brasil E-mail: iago.tavares@liga.org.br Igor Henrique Santos ORCID: https://orcid.org/0000-0003-1514-5475 Hospital Monsenhor Walfredo Gurgel, Brasil E-mail: igorhsantoscm@gmail.com

Lucas Ribeiro de Moraes Freitas ORCID: https://orcid.org/0000-0003-4276-8504 Universidade Federal da Paraíba, Brasil E-mail: lucasribeirodemf@gmail.com

Renner Cassio Nunes de Lucena ORCID: https://orcid.org/0000-0003-1605-6167 Universidade Federal do Rio Grande do Norte, Brasil E-mail: med.rennercassio@gmail.com

Vitor Luiz de Andrade Barbalho ORCID: https://orcid.org/0000-0001-5738-1789 Universidade de Pernambuco, Brasil E-mail: vitor.barbalho@upe.br

\begin{abstract}
Resumo
A neurofibromatose tipo 1 (NF1), consiste em uma doença genética. Quando comparada às outras duas formas, neurofibromatose tipo 2 (NF2) e schwannomatose, a NF1 é a apresentação mais comum. O quadro clínico geralmente se apresenta como máculas, sardas axilares e/ou inguinais, nódulos de Lisch (hamartomas de íris) e neurofibromas. O presente estudo trata-se de um relato de caso, o qual tem como objetivo relatar as repercussões e achados raros em uma idosa diagnosticada com NF1. A clínica da paciente começou há cerca de 45 anos quando buscou um serviço de saúde devido crises recorrentes de diarréia e hérnia em região inguinal. Foi solicitada uma USG de abdome total que evidenciou uma massa em região retropancreática característica de paraganglioma. Após 4 anos, a paciente possuía hipertensão primária de difícil controle com picos paroxísticos, sendo em seguida diagnosticado um feocromocitoma por meio de exames de imagem e exames laboratoriais. Durante o acompanhamento de rotina, uma ressonância magnética (RM) de abdome e pelve identificou nódulo sólido mantendo íntima relação com alças intestinais de delgado, que na imunohistoquímica tratava-se de um Tumor do Estroma Gastrointestinal (GIST). A paciente segue com acompanhamento oncológico na sua cidade e encontra-se sem queixas. Ultimamente tem apresentado lapsos de memória intermitentes e alguns episódios de quedas. Dessa forma, após uma revisão da literatura, descobriu-se que o tema em questão raramente é discutido, verificando-se escassos registros da existência simultânea dos três tumores citados, o que reforça, portanto, a importância deste estudo.
\end{abstract}

Palavras-chave: Neurofibromatose 1; Paraganglioma; Feocromocitoma; Tumores do estroma gastrointestinal. 


\begin{abstract}
Neurofibromatosis type 1 (NF1) is a genetic disease. When compared to the other two forms, neurofibromatosis type 2 (NF2) and schwannomatosis, NF1 is the most common presentation. The clinical manifestations usually present as macules, axillary and/or inguinal freckles, Lisch nodules (iris hamartomas) and neurofibromas. The present study is a case report, which aims to report the repercussions and rare findings in an elderly woman diagnosed with NF1. The patient's clinic started about 45 years ago when she sought a health service due to recurrent bouts of diarrhea and hernia in the inguinal region. A USG of the total abdomen was requested, which showed a mass in the retropancreatic region characteristic of a paraganglioma. After 4 years, the patient had difficult-to-control primary hypertension with paroxysmal peaks, and a pheochromocytoma was subsequently diagnosed by imaging and laboratory tests. During routine follow-up, magnetic resonance imaging (MRI) of the abdomen and pelvis identified a solid nodule maintaining a close relationship with small intestinal loops, which in immunohistochemistry was a Gastrointestinal Stromal Tumor (GIST). The patient continues with oncological follow-up in her city and has no complaints. She has lately been experiencing intermittent memory lapses and a few episodes of falling. Thus, after a review of the literature, it was found that the topic in question is rarely discussed, with few records of the simultaneous existence of the three mentioned tumors, which reinforces, therefore, the importance of this study.
\end{abstract}

Keywords: Neurofibromatosis 1; Pheochromocytoma; Paraganglioma; Gastrointestinal stromal tumors.

\title{
Resumen
}

La neurofibromatosis tipo 1 (NF1) es una enfermedad genética. Cuando se compara con las otras dos formas, neurofibromatosis tipo 2 (NF2) y schwannomatosis, NF1 es la presentación más común. El cuadro clínico suele presentarse como máculas, pecas axilares y/o inguinales, nódulos de Lisch (hamartomas del iris) y neurofibromas. El presente estudio es un reporte de caso, que tiene como objetivo relatar las repercusiones y hallazgos raros en una anciana diagnosticada con NF1. La clínica de la paciente comenzó hace unos 45 años cuando acudió a un servicio de salud debido a episodios recurrentes de diarrea y hernia en la región inguinal. Se solicitó USG de abdomen total, que mostró masa en región retropancreática característica de paraganglioma. A los 4 años presenta hipertensión primaria de difícil control con picos paroxísticos, siendo posteriormente diagnosticado de feocromocitoma por estudios de imagen y laboratorio. Durante el seguimiento de rutina, la resonancia magnética nuclear (RMN) de abdomen y pelvis identificó un nódulo sólido manteniendo una estrecha relación con asas de intestino delgado, que en inmunohistoquímica fue un Tumor del Estroma Gastrointestinal (GIST). La paciente continúa con seguimiento oncológico en su ciudad y no presenta quejas. Últimamente, ha tenido lapsos de memoria intermitentes y algunos episodios de caídas. Así, luego de una revisión de la literatura, se encontró que el tema en cuestión es poco discutido, con pocos registros de la existencia simultánea de los tres tumores mencionados, lo que refuerza, por tanto, la importancia de este estudio.

Palabras clave: Neurofibromatosis 1; Feocromocitoma; Paraganglioma; Tumores del estroma gastrointestinal.

\section{Introdução}

A neurofibromatose consiste em uma doença genética a qual pode existir de três formas distintas: tipo 1 (NF1), tipo 2 (NF2) e schwannomatose (Ciavarelli, 2021). A NF1, antes conhecida como doença de Von Recklinghausen, é o tipo mais frequente. Geneticamente, a NF1 possui caráter autossômico dominante e incidência de 1: 2.600 a 3.000 indivíduos (Evans et al., 2010).

A presença de variantes patogênicas no gene NF1 acarretam em ausência da produção ou deficiência da função da proteína, causando um amplo espectro de achados clínicos (Messiaen et al., 2008). As manifestações clínicas acontecem, geralmente, por meio da apresentação de máculas, sardas axilares e / ou inguinais, nódulos de Lisch (hamartomas de íris) e neurofibromas. Outras condições como escoliose, pseudoartrose tibial, displasia orbital, gliomas da via óptica e glioblastoma, neurofibromas espinhais, neurofibromas plexiformes e tumores malignos da bainha do nervo periférico, dificuldades de aprendizagem e déficits de atenção podem estar presentes em alguns indivíduos (Gutmann et al., 2017). O diagnóstico é clínico, principalmente avaliando o histórico familiar do paciente e o exame físico dos sistemas esquelético, neurológico e tegumentar.

Um ponto importante a ser ressaltado é que ainda não há um tratamento único para NF1. Apesar de já existir uma evolução com relação a compreensão dessa condição, alguns desafios ainda persistem; uma assistência multidisciplinar é necessária em prol do cuidado longitudinal para pessoas com NF1 (Blank et al., 2018). Partindo desse contexto, o presente 
Research, Society and Development, v. 11, n. 3, e3911326166, 2022

(CC BY 4.0) | ISSN 2525-3409 | DOI: http://dx.doi.org/10.33448/rsd-v11i3.26166

estudo se propõe relatar as repercussões e achados raros em uma idosa diagnosticada com NF1.

\section{Metodologia}

Este é um estudo de caso descritivo, observacional e retrospectivo, de acordo com Yin, 2015. O artigo foi desenvolvido a partir de um processo de avaliação de exame clínico, anamnese, exame físico e testes de imagem em associação com uma pesquisa bibliográfica sobre o assunto, utilizando como principais bases de dados Medline, Lilacs e Cochrane Library, os descritores "Neurofibromatose 1"; "Paraganglioma"; "Feocromocitoma"; "Tumores do Estroma Gastrointestinal". O estudo seguiu os preceitos éticos. Após autorização da direção clínica do hospital para a utilização dos registos médicos e a assinatura do Termo de Consentimento Livre e Esclarecido (ICF), foi realizado o relato do caso. A paciente recebeu esclarecimento sobre a garantia do sigilo das informações coletadas, tornando-se público apenas o caso, preservando o anonimato.

\section{Apresentação do Caso}

M.S., feminino, 45 anos de idade, buscou o serviço de saúde por crises recorrentes de diarréia e hérnia em região inguinal. Logo após a sua chegada solicitou-se uma Ultrassonografia (USG) de abdome total, que imediatamente evidenciou um tumor retropancreático. Posteriormente, ratificou-se que tal massa consistia em um paraganglioma. Destarte, realizou-se uma abordagem cirúrgica para correção da hérnia inguinal e em seguida o encaminhamento para um hospital de referência em oncologia do estado, visando a programação da exérese do tumor.

Durante a sua internação no serviço de referência, iniciou - se uma investigação mais ampla de todo o quadro da paciente. Seus sinais clínicos, como a presença de várias manchas “café com leite” e neurofibromas, permitiram estabelecer o diagnóstico de neurofibromatose tipo 1.

Após 4 anos da retirada do paraganglioma, a paciente possuía hipertensão primária de difícil controle e episódios paroxísticos de aumento pressórico, sendo em seguida diagnosticada, por meio de exames de imagem e exames laboratoriais, com a presença de feocromocitoma. Após isso, a paciente foi submetida a adrenalectomia unilateral direita, sendo então cessados os picos hipertensivos.

Nos exames de rotina para acompanhamento, uma ressonância magnética (RM) de abdome e pelve identificou nódulo sólido, lobulado e predominantemente homogêneo, mantendo íntima relação com alças intestinais de delgado e pequeno contato com a parede vesical de 4,3 x 3,4 x 2,6 cm. Em seguida, foi realizada enterectomia para retirada e análise desse nódulo, evidenciando, por conseguinte, a presença de neoplasia de células fusiformes. Após análise imunohistoquímica foi diagnosticado tumor gastrointestinal (GIST) KI $37=3 \%$.

Aos 66 anos de idade, a paciente continua sendo acompanhada pelo serviço de oncologia da sua cidade e encontra-se sem queixas, embora tenha apresentado ultimamente lapsos de memória intermitente e alguns episódios de quedas. A existência de alguns nódulos estáveis em mediastino superior e anterior evidenciados por Tomografias Computadorizadas (TC) de rotina (Imagem 1) não interferem na sua qualidade de vida. Além disso, ainda há a presença manchas café com leite (Imagem 2) e neurofibromas (Imagem 3) em região de membros inferiores, superiores, tórax e abdome (Imagem 4). 
Research, Society and Development, v. 11, n. 3, e3911326166, 2022

(CC BY 4.0) | ISSN 2525-3409 | DOI: http://dx.doi.org/10.33448/rsd-v11i3.26166

Imagem 1: Tomografía computadorizada de tórax evidenciando achados nódulares inespecíficos bilateralmente.

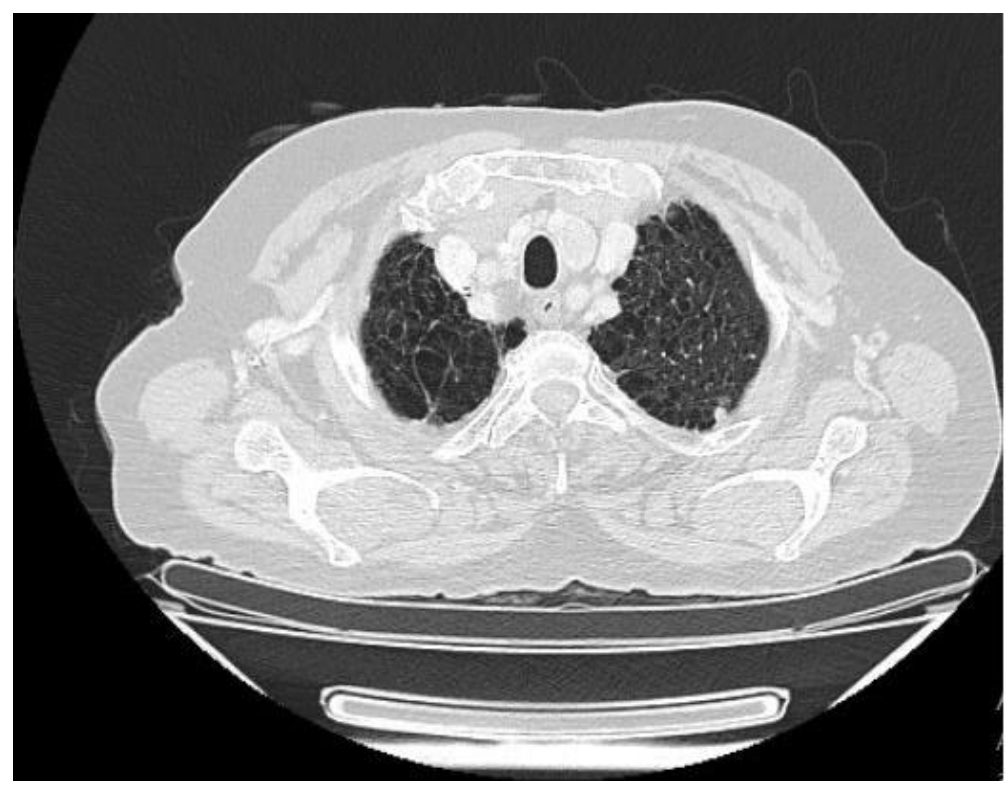

Fonte: Autores.

Imagem 2: Manchas "café com leite" na região de coxa esquerda. A presença de tais manchas espalhadas pelo corpo são critérios diagnósticos de Neurofibromatose tipo 1.

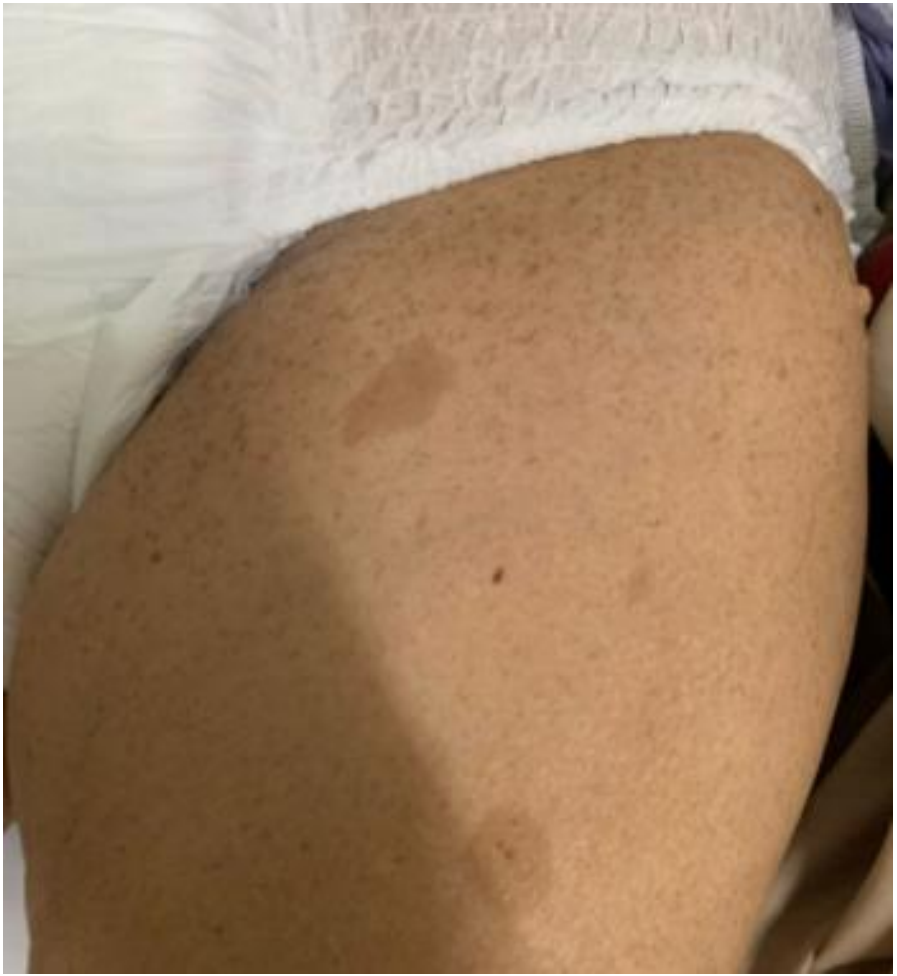

Fonte: Autores. 
Research, Society and Development, v. 11, n. 3, e3911326166, 2022

(CC BY 4.0) | ISSN 2525-3409 | DOI: http://dx.doi.org/10.33448/rsd-v11i3.26166

Imagem 3: Neurofibroma em membro superior direito. A presença de tais neurofibromas benignos configuram critérios diagnósticos para Neurofibromatose tipo 1.

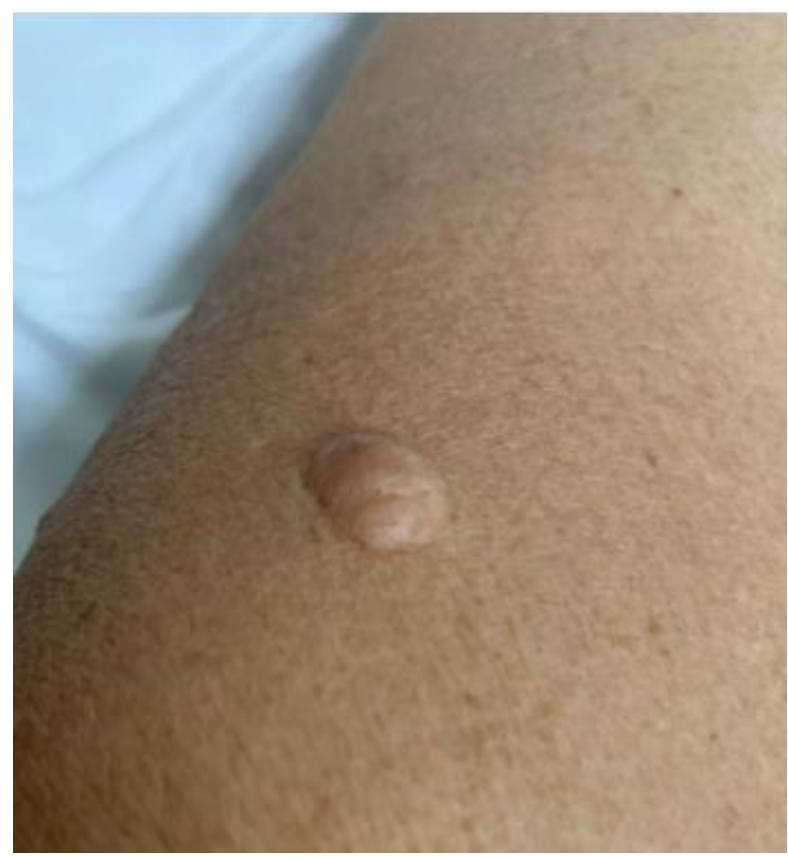

Fonte: Autores.

Imagem 4: Manchas café com leite e neurofibromas distribuídos em região torácica e abdominal.

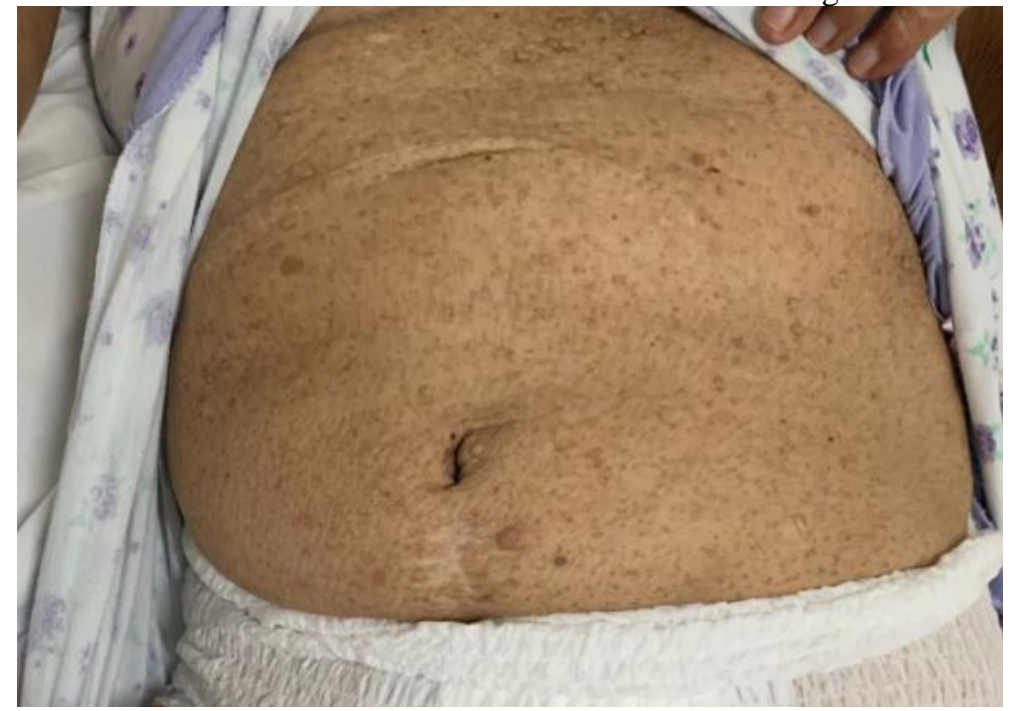

Fonte: Autores.

Por fim, é válido salientar a presença de um forte histórico familiar de neurofibromatose entre seus descendentes. A filha faleceu aos 22 anos de idade por complicações da neurofibromatose tipo $2 \mathrm{e}$, atualmente, o neto de 5 anos de idade, também possui diagnóstico com neurofibromatose do tipo schwannomatose.

\section{Discussão}

A Neurofibromatose tipo 1 (NF1) é uma doença genética autossômica dominante com uma incidência de aproximadamente 1:3000 indivíduos (Kallionpää et al., 2018; Reynolds, Browning, Nawroz, \& Campbell, 2003). A sua patogênese está relacionada a variantes patogênicas do gene NF1 localizado no cromossomo 17q11.2, o que resulta em perda 
Research, Society and Development, v. 11, n. 3, e3911326166, 2022

de produção ou função reduzida da proteína neurofibromina (Gutmann et al., 2017). A penetração, ou a probabilidade de o indivíduo portador da variante manifestar o distúrbio, é completa. No entanto, NF1 é altamente variável em sua expressão, ou seja, a gravidade e as manifestações específicas do transtorno variam entre os indivíduos afetados dentro da mesma família e de uma família para outra (Easton et al.,1993; Koczkowska et al., 2020).

Dessa forma, diversas manifestações clínicas podem ser encontradas em pacientes com NF1, como máculas café com leite, sardas axilares e inguinais, hamartomas de Íris (nódulos de Lish), anormalidades ósseas e a predisposição ao desenvolvimento de tumores benignos e malignos (Carvalho et al.,2021; Celik et al.,2021; Mahdi et al.,2020; Miraglia et al., 2020; Reynolds et al., 2003). Assim, destacamos a elevada prevalência de cânceres nesta população, sendo o risco de 5\% a 15\% maior do que a população geral, com idade de início mais precoce e pior prognóstico (Landry et al., 2021).

Entre os possíveis tumores benignos nessa população, os neurofibromas são os mais comuns, seguidos pelos gliomas das vias ópticas (OPGs). Tumores estromais gastrointestinais (GISTs), feocromocitomas e paragangliomas, presentes no caso relatado, são bem menos comuns (Alkhayyat et al., 2021; Al-Sharefi et al., 2019; Ylä-Outinen et al.,2019). Ademais, ao se analisar a combinação desses três tumores em pacientes com NF1, Gorgel et al. descreveram a existência de apenas 15 casos documentados na língua inglesa até o ano de 2014 (Gorgel et al., 2014). Sendo assim, reforça-se a raridade da apresentação da doença no nosso caso, visto que a coexistência de tais tumores é pouco descrita.

Os critérios de diagnóstico para NF1 desenvolvidos pela National Institutes of Health (NIH) dos Estados Unidos em 1987 e atualizados em 2021 (Legius et al., 2021) são altamente específicos e sensíveis, com 97 por cento das pessoas afetadas atendendo aos critérios de diagnóstico aos oito anos de idade e o restante atendendo aos critérios aos 20 anos de idade (DeBella et al.,2000). De maneira geral, tais critérios são baseados na presença das características clínicas mais comuns da doença, como a presença de máculas café com leite, neurofibromas, nódulos de Lisch, além de testes genéticos demonstrando variantes patogênicas do gene NF1.

Não existe um tratamento geral para neurofibromatose tipo 1. Portanto, o tratamento visa o manejo dos sintomas e das complicações, objetivando a melhora da qualidade de vida dos pacientes. A abordagem para o tratamento dos vários tumores associados a NF1 depende do tipo de tumor, seu efeito nos tecidos adjacentes e complicações relacionadas. Ademais, destacase a importância do acompanhamento multiprofissional para detecção precoce e tratamento sintomático das complicações à medida que elas surgem (Hirbe et al.,2014).

Quanto ao prognóstico, os dados de índice de sobrevida em pessoas com NF1 são limitados. Em um relatório usando dados de atestados de óbitos dos Estados Unidos de 1983 a 1997, a idade de morte foi menor em pacientes NF1 em comparação com a da população em geral (média de 54,5 contra 70,1 anos e mediana de 59 contra 74 anos, respectivamente) (Rasmussen et al.,2001). Ademais, a sobrevida específica para pacientes com NF1 que desenvolveram câncer é menor do que a de pacientes semelhantes sem NF1 com cânceres esporádicos (Landry et al., 2021).

Outrossim, vale ressaltar a importância do aconselhamento genético para o manejo da neurofibromatose tipo 1. Devido ao fato da NF1 ter um padrão de herança autossômica dominante, o aconselhamento deve ser fornecido aos pacientes e familiares, incluindo informações sobre a hereditariedade da doença, risco de recorrência em outros filhos, curso progressivo e prognóstico.

\section{Conclusão}

O relato evidencia um caso incomum e complexo de associação de tumores estromal gastrointestinal, feocromocitoma e paraganglioma em paciente portador de neurofibromatose tipo 1 (NF1). Para a realização do seu diagnóstico, faz-se uso de história clínica e exame físico dos sistemas esquelético, neurológico e tegumentar. Atualmente, embora haja uma evolução na 
Research, Society and Development, v. 11, n. 3, e3911326166, 2022 (CC BY 4.0) | ISSN 2525-3409 | DOI: http://dx.doi.org/10.33448/rsd-v11i3.26166

compreensão dos mecanismos da condição, ainda não existe um tratamento padrão para o NF1, apoiando-se na assistência multidisciplinar voltada para o cuidado longitudinal. Após uma revisão da literatura, descobriu-se que o tema em questão raramente é discutido, verificando-se escassos registros da existência simultânea dos três tumores. Logo, espera-se que o presente relato colabore com a ampliação do conhecimento sobre achados raros em pessoas com NF1, de forma a poder contribuir para o desenvolvimento científico de condições e equipamentos que promovam uma melhor assistência à saúde dessas pessoas.

\section{Referências}

Alkhayyat, M., Saleh, M. A., Coronado, W., Abureesh, M., Zmaili, M., Qapaja, T., ... Cooper, G. (2021). Epidemiology of neuroendocrine tumors of the appendix in the USA: A population-based national study (2014-2019). Annals of Gastroenterology, 34, 713-720.

Al-Sharefi, A., Javaid, U., Perros, P., Ealing, J., Truran, P., Nag, S., .. James, R. A. (2019). Clinical Presentation and Outcomes of Phaeochromocytomas/Paragangliomas in Neurofibromatosis Type 1. European Endocrinology, 15, 95-100.

Blank, P., Li, N., Ullrich, N. J., Fisher, M. J., Bhatia, S., Yasui, Y., ... Krull, K. R. (2018). Neurofibromatosis type 1 and risk of late outcomes after a primary tumor: A report from the Childhood Cancer Survivor Study. Journal of Clinical Oncology, 36, 10563-10563.

Carvalho, A. A., Martelli, D. R. B., Carvalho, M. F. A., Swerts, M. S. O., \& Júnior, H. M. (2021). Cafe-au-lait spots as a clinical sign of syndromes. Research, Society and Development, 10, e14310917607-e14310917607.

Celik, B., Aksoy, O. Y., Bastug, F., \& Poyrazoglu, H. G. (2021). Renal manifestations in children with neurofibromatosis type 1. European Journal of Pediatrics, 180, 3477-3482.

Ciavarelli, P. Y. (2021). Neurofibromatosis Tipo 1. Ediciones Servicop.

DeBella, K., Szudek, J., \& Friedman, J. M. (2000). Use of the national institutes of health criteria for diagnosis of neurofibromatosis 1 in children. Pediatrics, $105,608-614$.

Easton, D. F., Ponder, M. A., Huson, S. M., \& Ponder, B. A. (1993). An analysis of variation in expression of neurofibromatosis (NF) type 1 (NF1): Evidence for modifying genes. American Journal of Human Genetics, 53, 305-313.

Evans, D. G., Howard, E., Giblin, C., Clancy, T., Spencer, H., Huson, S. M., \& Lalloo, F. (2010). Birth incidence and prevalence of tumor-prone syndromes: Estimates from a UK family genetic register service. American Journal of Medical Genetics. Part A, 152A, $327-332$.

Gorgel, A., Cetinkaya, D. D., Salgur, F., Demirpence, M., Yilmaz, H., Karaman, E. H., ... Paker, I. (2014). Coexistence of Gastrointestinal Stromal Tumors (GISTs) and Pheochromocytoma in Three Cases of Neurofibromatosis Type 1 (NF1) with a Review of the Literature. Internal Medicine, 53, $1783-1789$.

Gutmann, D. H., Ferner, R. E., Listernick, R. H., Korf, B. R., Wolters, P. L., \& Johnson, K. J. (2017). Neurofibromatosis type 1. Nature Reviews. Disease Primers, 3, 17004 .

Hirbe, A. C., \& Gutmann, D. H. (2014). Neurofibromatosis type 1: A multidisciplinary approach to care. The Lancet. Neurology, 13, 834-843.

Kallionpää, R. A., Uusitalo, E., Leppävirta, J., Pöyhönen, M., Peltonen, S., \& Peltonen, J. (2018). Prevalence of neurofibromatosis type 1 in the Finnish population. Genetics in Medicine: Official Journal of the American College of Medical Genetics, 20, $1082-1086$.

Koczkowska, M., Callens, T., Chen, Y., Gomes, A., Hicks, A. D., Sharp, A., ... Messiaen, L. M. (2020). Clinical spectrum of individuals with pathogenic NF1 missense variants affecting p.Met1149, p.Arg1276, and p.Lys1423: Genotype-phenotype study in neurofibromatosis type 1. Human Mutation, 41, 299-315.

Landry, J. P., Schertz, K. L., Chiang, Y.-J., Bhalla, A. D., Yi, M., Keung, E. Z., ... Torres, K. E. (2021). Comparison of Cancer Prevalence in Patients With Neurofibromatosis Type 1 at an Academic Cancer Center vs in the General Population From 1985 to 2020. JAMA Network Open, 4 , e210945.

Legius, E., Messiaen, L., Wolkenstein, P., Pancza, P., Avery, R. A., Berman, Y., ... Plotkin, S. R. (2021). Revised diagnostic criteria for neurofibromatosis type 1 and Legius syndrome: An international consensus recommendation. Genetics in Medicine, 23, $1506-1513$.

Mahdi, J., Goyal, M. S., Griffith, J., Morris, S. M., \& Gutmann, D. H. (2020). Nonoptic pathway tumors in children with neurofibromatosis type 1. Neurology, 95, e1052-e1059.

Messiaen, L. M., \& Wimmer, K. (2008). NF1 Mutational Spectrum. Neurofibromatoses, 16, 63-77.

Miraglia, E., Moliterni, E., Iacovino, C., Roberti, V., Laghi, A., Moramarco, A., \& Giustini, S. (2020). Cutaneous manifestations in neurofibromatosis type 1. La Clinica Terapeutica, 171, e371-e377.

Rasmussen, S. A., Yang, Q., \& Friedman, J. M. (2001). Mortality in neurofibromatosis 1: An analysis using U.S. death certificates. American Journal of Human Genetics, 68, 1110-1118.

Reynolds, R. M., Browning, G. G. P., Nawroz, I., \& Campbell, I. W. (2003). Von Recklinghausen's neurofibromatosis: Neurofibromatosis type 1. The Lancet, $361,1552-1554$.

Ylä-Outinen, H., Loponen, N., Kallionpää, R. A., Peltonen, S., \& Peltonen, J. (2019). Intestinal tumors in neurofibromatosis 1 with special reference to fatal gastrointestinal stromal tumors (GIST). Molecular Genetics \& Genomic Medicine, 7, e927.

Yin, R. K. (2015). O estudo de caso. Bookman. 